\title{
Random Flight Model Analysis of Protein-Surfactant Complexes
}

\author{
Debasish Saha, ${ }^{1,3}$ Debes Ray, ${ }^{1}$ Joachim Kohlbrecher ${ }^{2}$ and Vinod K. Aswal ${ }^{1, \text { a) }}$ \\ ${ }^{1}$ Solid State Physics Division, Bhabha Atomic Research Centre, Mumbai 400 085, India \\ ${ }^{2}$ Laboratory for Neutron Scattering and Imaging, Paul ScherrerInstitut, CH-5232 PSI Villigen, Switzerland \\ ${ }^{3}$ Department of Science and Technology (DST), India \\ ${ }^{a)}$ Corresponding author: vkaswal@barc.gov.in
}

\begin{abstract}
The protein-surfactant complexes have a wide range of applications in the foods, cosmetics and pharmaceutical industry. Small angle neutron scattering (SANS) has been carried out to examine the interaction of bovine serum albumin (BSA) protein with sodium dodecyl sulfate (SDS) surfactant. The structure of protein-surfactant complexes is difficult to interpret due to the presence of different structure factor in such two-component systems. We have made the use of salt and optimize its concentration to minimize the contributions from structure factor arising from the charged inter-complex interaction. The results are analyzed by the random flight model representing a beads-on-string type structure for the protein-surfactant complex. The introduction of the random flight model successfully resolves some serious issues of the previously used fractal model for protein-surfactant complexes. In the modeled complex, the unfolded protein wraps around number of micelles to form a cluster. The micelles get elongated with increasing concentration of the surfactant while the number of micelles per cluster is decreased. The pathway of protein unfolding is described in terms of the changes in the micellar size, the number of micelles formation per cluster, the separation between the micelles in the cluster, the aggregation number of micelles and the number of proteins per cluster.
\end{abstract}

\section{INTRODUCTION}

Proteins and surfactants both are surface active materials and important entities in soft material research which have several interesting applications [1]. There are different models for the structure of protein-surfactant complexes. In one of the most commonly used fractal model, protein-surfactant complexes represent a beadnecklace structure of micelle-like clusters adsorbed along the unfolded protein chain [2-4]. However, the absence of correlation hole in the fractal model makes it unphysical for protein-surfactant complexes and more applicable for the polydisperse systems, where the correlation hole can be smeared out. In addition to that, the fractal model does not include the scattering from the protein, which limits its use to explain the scattering from protein-surfactant complexes on the absolute scale. Therefore, we have considered another model called the random flight model, which represents a beads-on-a-string like structure of protein-surfactant complexes $[5,6]$. The random flight model considers the scattering from individual micelles and the overall cluster as well. In order to validate this model for such protein-surfactant complexes, the inter-complex interaction should be minimized. The optimization of salt concentration can screen the structure factor arising from the inter-complex interaction. The other way to minimize the inter-complex structure factor is the infinite dilution of the system. However, the dilution of the system is not a good idea for such systems as it decreases the surfactant concentration below Critical Aggregation Concentration (CAC) and disrupting the micelle formation.

The unfolding of BSA protein in presence of SDS surfactant is examined and the data are explained in terms of the random flight model. To simplify the analysis, the inter-complex interaction is minimized by adding salt in the protein-surfactant complexes. As the SDS concentration is increased, the protein-decorated micelles start elongating, and therefore, the number of micelles per cluster is decreased while the separation between the centers of the micelles is increased. The detailed structural analysis of the protein-surfactant complexes reveals that the unfolded protein wraps the large micelles through its surface. 


\section{EXPERIMENTAL}

BSA with 99\% purity and MW = $66 \mathrm{kDa}$ was purchased from Sigma-Aldrich. The protein was received as lyophilized powder and used without any purification. Anionic SDS and $\mathrm{NaCl}$ were purchased from Sigma-Aldrich. $\mathrm{D}_{2} \mathrm{O}\left(99.9\right.$ atom $\%$ D) was used instead of $\mathrm{H}_{2} \mathrm{O}$ to ensure good contrast for SANS experiments. All the samples were dissolved in $20 \mathrm{mM}$ phosphate buffer at $\mathrm{pH}=7.0$ at variable salt concentrations. SANS experiments were carried out on the SANS-I facility at Swiss Spallation Neutron Source SINQ, Paul Scherrer Institute, Switzerland [7]. All the measurements were performed at $\lambda=8 \AA$ with the wavelength resolution $(\Delta \lambda / \lambda)$ about $10 \%$. The raw data were radial averaged, corrected for electronic background and empty cell, and normalized to an absolute cross-sectional unit using standard procedure.

\section{DATA ANALYSIS}

The differential scattering cross-section per unit volume $(\mathrm{d} \Sigma / \mathrm{d} \Omega)$ as a function of $q$ for monodisperse interacting particles is given by

$$
\left(\frac{d \Sigma}{d \Omega}\right)(q)=N P(q) S(q)+B
$$

where $N$ is the particle number density. $P(q)$ and $S(q)$ are the form factor and structure factor, respectively, and $B$ is the incoherent background coming from the hydrogen content in different components. In the present system, the form factor of a micelle, is assumed to be prolate ellipsoidal core-shell structure. $P(q)$ of prolate ellipsoidal particle with semi-minor axis $R$ and axial anisotropy $\varepsilon$ can be written as

$$
P_{e c s}(q)=\left(\rho_{p}-\rho_{s}\right)^{2} V^{2} \int_{0}^{\pi / 2} F[q, r(R, \varepsilon, \beta)]^{2} \sin \beta d \beta
$$

where $F(q, r)=\frac{3[\sin (q r)-q r \cos (q r)]}{(q r)^{3}}$ and $r(R, \varepsilon, \beta)=R\left(\sin ^{2} \beta+\varepsilon^{2} \cos ^{2} \beta\right)^{1 / 2}$

with $\rho_{\mathrm{p}}$ and $\rho_{\mathrm{s}}$ are respectively the scattering length densities of the particle and solvent. $V$ is the volume of the particle. $\beta$ is the angle between the directions of semi-major axis and wave vector transfer.

The structure factor of the random flight model with a constant step size can be expressed as[8]

$$
S(q)=\left[\frac{2}{1-\sin (q D) /(q D)}-1-\frac{2\left[1-(\sin (q D) /(q D))^{N_{C L U}}\right]}{N_{C L U}(1-\sin (q D) /(q D))^{2}} \frac{\sin (q D)}{q D}\right]
$$

where $N_{\mathrm{CLU}}$ is the random flight steps (number of micelles per cluster) and $D$ is the step size (distance between the centers of two micelles). $N_{\mathrm{CLU}}$ is calculated as the weighted linear combination of [ $\left.N_{\mathrm{CLU}}\right]$ and $\left[N_{\mathrm{CLU}}\right]+1$, with [ $\left.N_{\mathrm{CLU}}\right]$ largest integer smaller than $N_{\mathrm{CLU}}$. The differential scattering cross-section per unit volume $(\mathrm{d} \Sigma / \mathrm{d} \Omega)$ for micelles with random-flight structure has been extracted by combining Eqs. 1-3 and using the decoupling approximation and can be expressed as [9]

$$
\left(\frac{d \Sigma}{d \Omega}\right)(q)=N_{m i c}\left[\left\langle F^{2}(q)\right\rangle+\langle F(q)\rangle^{2}(S(q)-1)\right]+B
$$

where $N_{\text {mic }}$ is the number density of micelles and calculated by $N_{\text {mic }}=N_{\mathrm{A}} \times\left(C_{\text {tot }}-C_{\mathrm{CAC}}\right) / \mathrm{N}_{\mathrm{agg}} . \mathrm{N}_{\mathrm{A}}$ is the Avogadro number, $C_{\text {tot }}$ is the total surfactant concentration, and $C_{\mathrm{CAC}}$ is the critical aggregation concentration of the surfactant.

\section{RESULTS AND DISCUSSION}

SANS measurements are carried out for pure BSA and SDS, and BSA with SDS (1wt \% each) in the presence of varying salt concentration $(0$ to $0.3 \mathrm{M} \mathrm{NaCl})$. The scattering intensities depend on the form factor and structure factor of the components. The presence of salt can significantly influence each of these factors and hence the change in the scattering data. We have added salt to the system to minimize the contributions of the structure factor for protein-surfactant complexes from inter-complex interaction [10]. 

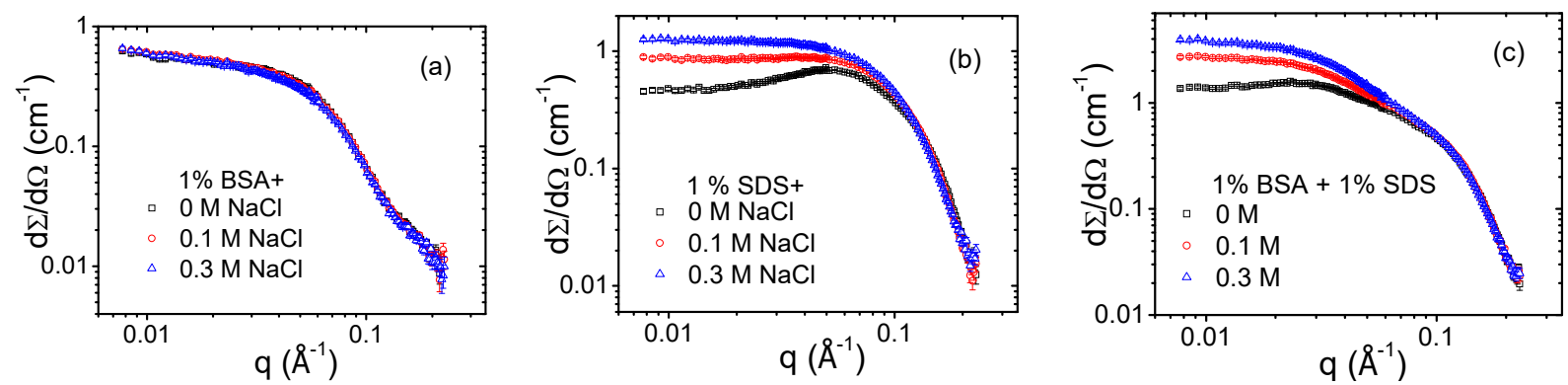

FIGURE 1. SANS data of (a) $1 \mathrm{wt} \%$ BSA (b) $1 \mathrm{wt} \%$ SDS and (c) $1 \mathrm{wt} \% \mathrm{BSA}+1 \mathrm{wt} \%$ SDS for varying salt concentration from 0 to $0.3 \mathrm{M} \mathrm{NaCl}$ at $\mathrm{pH}=7$ in $\mathrm{D}_{2} \mathrm{O}$.

No interparticle interaction is observed in the scattering data of $1 \mathrm{wt} \%$ BSA protein at different $\mathrm{NaCl}$ concentration $(S(q)=1)$ (Figure 1a). This is clear from the fact that scattering data do not show any noticeable change in presence of salt. The calculated form factor and the structure factor at different salt concentration are presented in Figure 2a. The form factor and structure factor obtained at different salt concentrations are same. On the contrary, a clear change in the scattering intensities of $1 \mathrm{wt} \%$ SDS is observed at different salt concentrations (Figure 2b). Initially, without salt, a repulsive structure factor is observed which lowers the scattering intensity in the low- $q$ range. The structure factor is suppressed by adding $0.3 \mathrm{M} \mathrm{NaCl}$, which can be observed form the flat scattering intensity in the low- $q$ range (Figure 1b). The similar behavior is observed for BSA-SDS complexes, where the inter-complex structure factor is completely suppressed at $0.3 \mathrm{M} \mathrm{NaCl}$ concentration (Figure 1c). However, the micelle formation by pure SDS and BSA-SDS are totally different, which reveals the strong interaction between these two entities. The salt concentration of $0.3 \mathrm{M} \mathrm{NaCl}$ was kept fixed in all the experiments for examining the interaction of BSA protein with SDS surfactant.
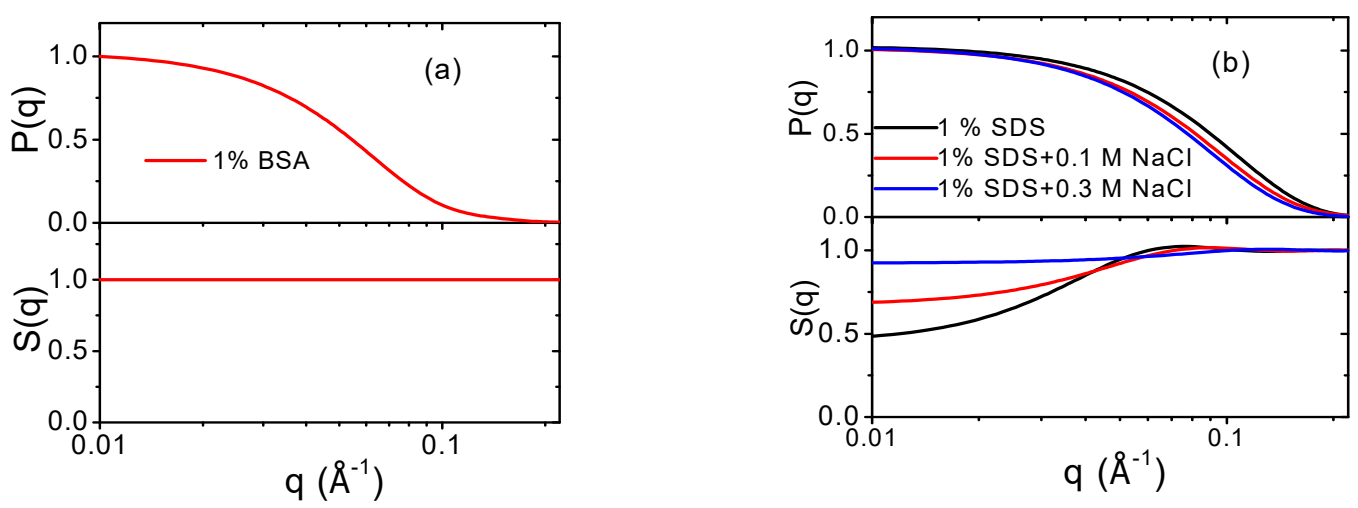

FIGURE 2. The form factor (top) and the structure factor (bottom) of (a) $1 \mathrm{wt} \%$ BSA and (b) $1 \mathrm{wt} \%$ SDS for varying salt concentration from 0 to $0.3 \mathrm{M} \mathrm{NaCl}$ at $\mathrm{pH}=7$ in $\mathrm{D}_{2} \mathrm{O}$.

In the next step, SANS measurements are carried out for BSA with anionic SDS at varying concentrations while keeping the salt concentration fixed at $0.3 \mathrm{M}$. The concentration of SDS is varied from 0 to $2 \mathrm{wt} \%$. The scattering features of the BSA-SDS complexes at different SDS concentrations significantly different from each other (Fig. 3a). The Indirect Fourier Transformation (IFT) of SANS data shows the appearance of the second maximum, which indicates the binding of the number of micelles to the protein in the resultant complexes (Figure $3 \mathrm{~b}$ ). The peak at lower- $r$ is related to the intra-micelle scattering and hence the size of the micelles, whereas the peak at higher- $r$ comes from the inter-micellar interaction within the complex and the center-to-center distance of one micelle to the next nearest micelle. In addition, the complex size also increases systematically with increasing SDS concentration $\left(D_{\max }\right.$ in Table 1). Thus, a higher surfactant to protein ratio enables the extension of the complex.

The detailed modeling of SANS data obtained from BSA-SDS complexes at different concentrations is shown in Figure 3a (solid lines are fitted curves). Due to the low CAC of SDS $(2.2 \mathrm{mM})$, they have displayed strong hydrophobic interaction with BSA through micelle formation. The random flight model analysis of SANS data depicts that the micelle size, micellar anisotropy $(\varepsilon)$, and the aggregation number increased with increasing the 
surfactant concentration and thus enhance the denaturation of BSA. On the other hand, the number of proteins per cluster is decreased while the distance $D$ increased with increasing the surfactant concentration. The analysis shows that one to three micelles can be presented in each cluster $\left(N_{\mathrm{CLU}}=1.6,1.9\right.$, and 2.2). However, the presence of the second maximum in the $p(\mathrm{r})$ function rules out the merger of small micelles to a single elongated one.
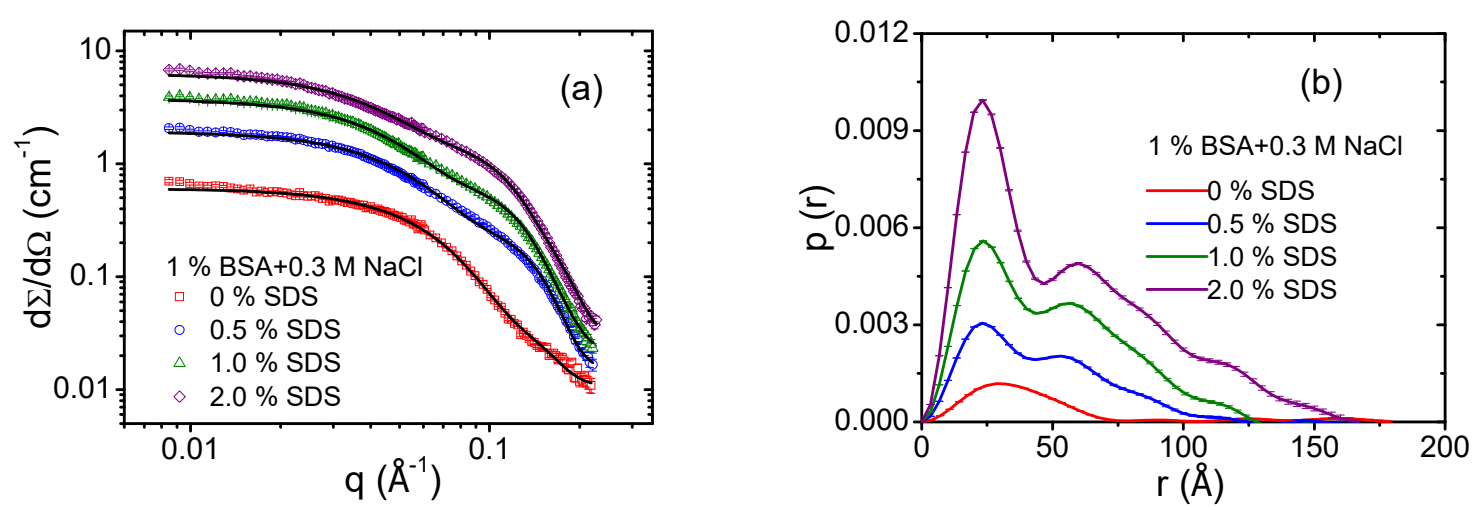

FIGURE 3. (a) SANS profiles of $1 \mathrm{wt} \%$ BSA in the presence of 0 to $2.0 \mathrm{wt} \%$ SDS and (b) Pair distance distribution functions $p(r)$ obtained from the fits of the experimental data.

TABLE 1: Fitted parameters of SANS data of protein-surfactant systems ( $1 \mathrm{wt} \% \mathrm{BSA}+\mathrm{c} w \mathrm{t} \% \mathrm{SDS})$ characterized by random flight model representing a beads-on-string like structure.

\begin{tabular}{cccccccc}
\hline $\boldsymbol{c}(\mathbf{w t} \%)$ & $\boldsymbol{D}_{\max }(\AA)$ & $\boldsymbol{R}(\AA)$ & $\boldsymbol{\varepsilon}$ & $\boldsymbol{N}_{\mathbf{C L U}}$ & $\boldsymbol{D}(\AA)$ & $\boldsymbol{N}_{\text {agg }}$ & $\mathbf{N}_{\text {prot }}$ cluster \\
\hline 0.5 & 124 & $17.8 \pm 0.1$ & $1.74 \pm 0.06$ & $2.2 \pm 0.1$ & $50 \pm 2$ & 118 & 2.6 \\
1.0 & 131 & $17.8 \pm 0.1$ & $2.24 \pm 0.07$ & $1.9 \pm 0.1$ & $59 \pm 3$ & 150 & 1.5 \\
2.0 & 160 & $17.3 \pm 0.1$ & $2.60 \pm 0.07$ & $1.6 \pm 0.1$ & $69 \pm 4$ & 160 & 1.1 \\
\hline
\end{tabular}

\section{CONCLUSION}

The validation of the random flight model in the structural analysis of BSA-SDS complexes from the SANS data has been carried out. The addition of salt screens the inter-complex structure factor and simplifies the modeling of the intra-complex structure formed by BSA-SDS. The unfolded protein binds to the surfactant micelles and wraps the protein-surfactant cluster through the surface. The micelles get elongated whereas the number of micelles per cluster is decreased in the pathway of protein unfolding.

\section{REFERENCES}

1. C. Sun, J. Yang, X. Wu, X. Huang, F. Wang, S. Liu, Biophys. J. 88, 3518-3524 (2005).

2. S.-H. Chen, J. Teixeira, Phys Rev Lett. 57, 2583-2586 (1986).

3. X.H. Guo, N.M. Zhao, S.H. Chen, J. Teixeira, Biopolymers. 29, 335-346 (1990).

4. S. Chodankar, V.K. Aswal, J. Kohlbrecher, R. Vavrin, A.G. Wagh, J. Phys. Condens. Matter. 19, 326102 (2007).

5. L. Giehm, C.L.P. Oliveira, G. Christiansen, J.S. Pedersen, D.E. Otzen, J. Mol. Biol. 401, 115-133 (2010).

6. D. Saha, D. Ray, J. Kohlbrecher, V.K. Aswal, ACS Omega. 3, 8260-8270 (2018).

7. J. Kohlbrecher, W. Wagner, J. Appl. Crystallogr. 33, 804-806 (2000).

8. The statistics of stiff chain molecules I. The particle scattering factor, Proc. R. Soc. Lond. Math. Phys. Eng. Sci. 316, 185-199 (1970).

9. J.S. Pedersen, Adv. Colloid Interface Sci. 70, 171-210 (1997).

10. S. Kumar, V.K. Aswal, J. Kohlbrecher, Langmuir. 28, 9288-9297 (2012). 